\title{
Progress in mesenchymal stem cell-based therapy for acute liver failure
}

\author{
Yong-Hong Wang, Dong-Bo Wu, Bing Chen, En-Qiang Chen * (1D and Hong Tang*
}

\begin{abstract}
Acute liver failure is a life-threatening clinical syndrome characterized by rapid development of hepatocellular necrosis leading to high mortality and resource costs. Numerous treatment strategies for acute liver failure simply prevent complications and decelerate disease progression. The only curative treatment for acute liver failure is liver transplantation, but there are many restrictions on the application of liver transplantation. In recent years, a growing number of studies have shown that stem cells can effectively treat acute liver failure. Several types of stem cells have been used to study liver diseases; mesenchymal stem cells are most commonly used because they are easy to obtain and present no ethical problems. The aims of this article are to review the current knowledge regarding therapeutic mechanisms of mesenchymal stem cells in acute liver failure, to discuss recent advancements in preclinical and clinical studies in the treatment of mesenchymal stem cells, and to summarize the methodological improvement of mesenchymal stem cell transplantation in treating liver failure.
\end{abstract}

Keywords: Mesenchymal stem cells, Acute liver failure, Treatment

\section{Background}

Acute liver failure (ALF) is a life-threatening clinical syndrome characterized by rapid hepatocellular necrosis due to various acute injuries induced by hepatotoxic drugs, immune-mediated attack, or viral infections. Notwithstanding the low morbidity of ALF, approximately one and six cases per million individuals annually worldwide, this serious disease will lead to high mortality and resource costs $[1,2]$. Currently, many ALF treatment strategies are aimed at simply preventing

\footnotetext{
* Correspondence: chenenqiang1983@hotmail.com; htang6198@hotmail.com Center of Infectious Diseases, West China Hospital of Sichuan University, Chengdu 610041, China
}

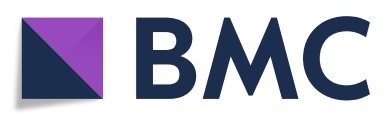

(c) The Author(s). 2018 Open Access This article is distributed under the terms of the Creative Commons Attribution 4.0 International License (http://creativecommons.org/licenses/by/4.0/), which permits unrestricted use, distribution, and reproduction in any medium, provided you give appropriate credit to the original author(s) and the source, provide a link to the Creative Commons license, and indicate if changes were made. The Creative Commons Public Domain Dedication waiver (http://creativecommons.org/publicdomain/zero/1.0/) applies to the data made available in this article, unless otherwise stated. complications and decelerating disease progression. The only curative treatment for ALF is liver transplantation, but there are many restrictions on the application of liver transplantation because of financial considerations, a shortage of donor livers, and immunosuppression-related complications [3]. Therefore, novel therapeutic methods for patients with ALF are urgently required. In recent years, a growing number of studies have shown that stem cells can effectively treat ALF.

Several types of stem cells, including embryonic stem cells, mesenchymal stem cells (MSCs), induced pluripotent stem cells, hepatic progenitor cells, and hematopoietic stem cells, have been used to study liver diseases [4]; however, MSCs are most commonly used because they are easy to obtain and present no ethical problems [5]. MSCs have the capacity of multiple differentiations and self-renewal and have been proven to be able to differentiate into a series of cell lineages, such as adipocytes, osteoblasts, chondroblasts, and hepatocyte-like cells (HLCs). In addition, they have other properties, including antiinflammatory effects, anti-apoptosis, immunosuppression, angiogenesis, promotion of tissue repair, and growth factor production. Although there is a great deal of preclinical and clinical research about MSCs in the treatment of ALF, the exact mechanism underlying the therapeutic role of MSCs remains unclear [6]. The aims of this review are to review the current knowledge regarding therapeutic mechanisms of MSCs in ALF, to discuss recent advancements in preclinical and clinical studies in the treatment of MSCs, and to summarize the method improvement of MSC transplantation in treating liver failure.

\section{MSCs differentiate into hepatocyte-like cells}

The idea of using hepatocytes to treat liver failure depends on a simple hypothesis that liver function can be improved by supplementing exogenous hepatocytes. Although liver failure can be treated via hepatocyte transplantation, it also faces multiple problems comprising the shortage of high-quality hepatocytes sources, rejection of 
allogeneic transplants, difficulty to expand, and losing hepatic characteristics in vitro [7, 8]. Previous studies have proven that rodent and human MSCs can differentiate into HLCs in vitro and in vivo, which is one of the mechanisms of MSCs in the treatment of liver failure. Therefore, MSCs may help resolve issues restricting the application of hepatocyte transplantation. Different groups have established several protocols to induce the differentiation of MSCs into HLCs in vitro. MSCs, such as bone marrow (BM-MSCs), adipose tissue (AT-MSCs), umbilical cord (UC-MSCs), and amniotic fluid (AF-MSCs), have been shown to differentiate into HLCs [9-12]. Human BM-MSCs are induced into HLCs via a serum-free maintenance medium of human hepatocyte supplemented with hepatocyte growth factor (HGF) and epidermal growth factor (EGF), which has been proven to retain the qualities of hepatocytes after their regional integration in mouse livers [9]. Differentiation of BM-MSCs and UC-MSCs was induced by differentiation medium supplemented with HGF, basic fibroblast growth factor (bFGF), and nicotinamide, followed by treatment with maturation medium containing dexamethasone, oncostatin M (OSM), and insulin-transferrin-selenium (ITS) [10]. AT-MSCs can differentiate into functional HLCs in vitro via culturing in a hepatic culture medium containing EGF, HGF, FGF1, FGF4, ITS, OSM, and dexamethasone. Meanwhile, AT-MSC-derived hepatocytes can be incorporated into the host liver and improve liver functions [11]. AF-MSCs were serumdeprived for $2 \mathrm{~d}$ in culture medium supplemented with EGF and bFGF. Differentiation was induced by treating AF-MSCs with differentiation medium containing HGF, bFGF, and dimethyl sulfoxide (DMSO) for $7 \mathrm{~d}$, followed by maturation medium comprising OSM, dexamethasone, and ITS for 2 weeks [12]. Demethylation reagents such as 5-azacytidine have been proven to be useful in inducing MSCs to differentiate into HLCs [9, 13, 14]. Although 5-azacytidine has been applied in clinical treatment of hematologic diseases [15], the side effects, including thrombocytopenia, myelosuppression, and pneumonia, are obvious [16]. Therefore, we should pay attention to its side effects when we use demethylation agents to induce differentiation. HLCs differentiated from different MSC types can be identified via various methods, including observation of hepatocyte-specific morphology, expression of hepatocyte-specific marker genes, and the functions of hepatocytes comprising glycogen storage, albumin production, uptake of lowdensity lipoprotein, indocyanine green uptake assay, urea secretion, and cytochrome P450 activity.

Many studies have shown that transplanted MSCs can directly differentiate into HLCs in vivo. Human UC-MSCs entered the injured liver induced by D-galactosamine/lipopolysaccharide (D-GalN/LPS) in mice and differentiated into HLCs, identified via positive staining of albumin
(ALB), alpha fetoprotein (AFP), and cytokeratin 18 (CK18) $[17,18]$. The transplantation of human UC-MSCs significantly improves the survival rate of acute hepatic necrosis rats induced by carbon tetrachloride $\left(\mathrm{CCl}_{4}\right)$. The underlying mechanisms may involve human UC-MSC trans-differentiation into HLCs and targeted migration to liver injury sites $[19,20]$. However, some experiments have suggested that MSCs cannot directly differentiate into HLCs in vivo. Xiao et al. showed that transplanted MSCs have no obvious evidence of hepatocyte trans-differentiation, upon assessing the survival, distribution, and hepatocyte markers of MSCs in vivo [21]. Chen et al. proved that AT-MSCs did not differentiate into hepatocytes after engrafting to livers within $3 \mathrm{~d}$ [22].

At present, treatment alternatives for liver failure between undifferentiated MSCs and HLCs are still controversial. Zagoura et al. reported that the effect of undifferentiated MSCs is better than that of HLCs, showing that AF-MSC-derived HLCs, compared with AF-MSCs and hepatic progenitor-like cells, failed to enter the damaged liver and contribute to recovery [12]. Similar results were obtained by Wang et al., who observed that HLCs expressed lower levels of $\mathrm{HGF}$ and were accompanied by impaired immunosuppression compared with MSCs. Therefore, undifferentiated MSCs may be more suitable than HLCs to treat liver diseases [23]. However, several recent studies have shown that the treatment effects of undifferentiated MSCs and HLCs are similar in ALF [24-27]. Undifferentiated MSCs and HLCs from adipose tissue, bone marrow, and the umbilical cord transplanted in a mouse model of acute fulminant hepatitis were equally able to regenerate injured liver tissue and save almost all of the mice $[24,26]$. Similarly, Li et al. found that uninduced BM-MSCs and HLCs had similar effects on the treatment of ALF in rats. Levels of alanine transaminase (ALT), aspartate transaminase (AST), and total bilirubin (TBIL) in the transplantation group were significantly higher than those in the control group and decreased significantly $7 \mathrm{~d}$ after transplantation [25]. No studies have reported better therapeutic effects of HLCs than undifferentiated MSCs, based on literature reviews. Therefore, hepatocyte-like differentiation may not be necessary for MSCs to treat liver failure.

\section{Mechanisms of MSC-mediated immunomodulation}

Most previous studies have shown that the therapeutic effects of MSCs in liver failure are potentially based on its release of trophic and immunomodulatory factors. Although the immunomodulatory mechanism of MSCs remains to be elucidated, they are likely to regulate immune cells by secreting soluble factors and intercellular contacts. MSCs can regulate adaptive and innate immune responses by inhibiting $\mathrm{T}$ cells and dendritic cells, reducing the activation and proliferation of $\mathrm{B}$ cells, 
promoting the production of regulatory $\mathrm{T}$ (Treg) cells, and inhibiting the proliferation and cytotoxicity of natural killer (NK) cells [28-33]. When MSCs play an immunoregulatory role, transforming growth factor-beta (TGF- $\beta$ ) and interleukin 10 (IL-10) are key factors regulating numerous inflammatory cells. Fang et al. showed that the levels of TGF- $\beta$ and IL-10 in serum increased significantly after injecting UC-MSCs but that the levels of IL-6, tumor necrosis factor-alpha (TNF- $\alpha$ ), and CD8 ${ }^{+}$ $\mathrm{T}$ cells in peripheral blood decreased significantly, which resulted in the repair of liver injury and improved disease developing and mortality rates [29]. Meanwhile, BM-MSCs can induce transient T-cell apoptosis through the Fas ligand (FasL)-dependent Fas pathway, and apoptotic $\mathrm{T}$ cells subsequently trigger macrophages to produce high levels of TGF- $\beta$, which leads to the upregulation of Treg cells to induce immune tolerance [28]. In fulminant hepatic failure (FHF), the therapeutic effects of MSCs are achieved primarily by reducing hepatic $\mathrm{CD}_{4}^{+} \mathrm{T}$-cell infiltration and activation, inhibiting $\mathrm{T}$ helper 1 (Th1) cells, and inducing Treg cells. Moreover, MSCs can induce a distinct liver population of $\mathrm{CD} 11 \mathrm{c}^{+} \mathrm{MHCII}{ }^{\mathrm{hi}} \mathrm{CD} 80^{\mathrm{lo}} \mathrm{CD} 86^{\text {lo }}$ regulatory dendritic cells that induced Treg cell differentiation through TGF- $\beta$ production [30]. It has been reported that BM-MSC infusion could improve the immunoregulatory activity by inhibiting liver NKT cells and this inhibition is systemic, not limited to the liver [33]. MSCs can inhibit cytotoxic $\mathrm{CD}^{+} \mathrm{T}$ lymphocyte (CTL) and NK cells through intercellular contact and paracrine factors such as indoleamine 2,3-dioxygenase (IDO), TGF- $\beta$, and prostaglandin $\mathrm{E}_{2}\left(\mathrm{PGE}_{2}\right)$ [3]. Of note, TGF- $\beta$ is a two-edged sword with immunosuppressive effects to alleviate liver inflammation $[3,28,30]$ but can also promote the progression of liver fibrosis [34, 35]. The immunomodulatory effect of MSCs on transforming the body into an antiinflammatory state is achieved by upregulating antiinflammatory Treg cells and reducing Th1 and Th17 cells in FHF. In addition, establishment of the antiinflammatory state after MSC transplantation may be indirectly induced via upregulation of M2-type macrophages, which secrete various anti-inflammatory factors, including chemokine ligand 1 (CCL-1) and IL-10, which upregulate Th2 and Treg cells [36]. In addition, MSCs can reduce B-cell proliferation through cell-cell contact and secretion of soluble factors [31]. Intravenous injection of MSCs alleviates acute hepatitis and NKT cell hepatotoxicity in an IDO-dependent paracrine manner; however, MSCs did not distinctly alter the total number of neutrophils producing IL-17, $\mathrm{CD}_{4}^{+}$, and $\mathrm{CD}^{+} \mathrm{T}$ lymphocytes in the injured liver [32]. Furthermore, MSC transplantation can effectively ameliorate liver injury in ALF rats by reducing the number and activity of neutrophils in both peripheral blood and the liver [37].

\section{The application of MSC-derived conditioned medium}

Recently, MSC-conditioned medium (MSC-CM) reportedly had similar therapeutic effects on the treatment of liver failure with MSC transplantation, and the therapeutic effects of MSC-CM may be the combined effect of free soluble factors and exosomes because both of them have been proven to be effective in treating liver failure. Lotfinia et al. reported that MSC-CM can improve liver function but not increase survival rate. In their study, MSC-CM could significantly enhance the viability of primary hepatocytes and increase the secretion of anti-inflammatory IL-10 from human blood mononuclear cells. Meanwhile, the biochemical and histopathological parameters of liver injury were improved $48 \mathrm{~h}$ after injection of MSC-CM; however, the survival rate of ALF mice was not increased 1 week after injection [38]. However, many studies have reported that MSC-CM could provide a significant survival benefit in FHF and promote the repair of damaged liver tissue by inhibiting apoptosis in hepatocytes, improving liver regeneration, and reducing panlobular leukocytic infiltrates [22, 36, 39-41]. MSC-CM contains numerous soluble factors associated with the survival benefits of FHF [12, 22, 40, 42]. MSC-CM treatment significantly reduced serum interferon-gamma (IFN- $\gamma$ ), IL-1 $\beta$, and IL-6 levels and elevated serum IL-10 levels compared with the control group. Proteomic analysis of MSC-CM showed that IL-10 levels increased most significantly in anti-inflammatory factors. Phosphorylation of signal transducer and activator of transcription 3 (STAT3) was upregulated after IL-10 infusion and AG490-induced STAT3 inhibition reversed the therapeutic effects of IL-10 [42]. Moreover, MSC-CM with high levels of HGF and vascular endothelial growth factor can improve the survival rate of ALF rats [22]. Previous studies indicated that conditioned medium derived from different cells has diverse therapeutic effects in liver failure. Conditioned medium of hepatic progenitor-like cells (HPL-CM) is more effective than conditioned medium from AF-MSCs in treating liver failure. Proteomic analysis showed that HPL-CM contained anti-inflammatory factors, including IL-10, IL-1-receptor antagonist (IL-1ra), IL-13, and IL-27, which could induce liver recovery [12]. Huang et al. observed that MSCs had a better therapeutic effect in FHF than MSC-CM by reducing macrophage infiltration into the damaged liver. In contrast, MSC-CM had a better inhibitory effect on fibrogenesis and necroinflammation in chronic liver injury by inhibiting the activation of hepatic stellate cells, promoting liver regeneration, and reducing hepatocyte apoptosis [36].

Notably, conditioned medium can also play a therapeutic role in liver failure via exosomes. Chen et al. found that treatment with menstrual blood stem cell-derived 
exosomes (MenSC-Ex) before D-GalN/LPS injection could reduce TNF- $\alpha$, IL- 6 , and IL- $1 \beta$ levels in circulation of FHF mice, inhibit hepatocyte apoptosis, improve liver function, and ultimately reduce the mortality of FHF mice [43]. Furthermore, MSCs can induce hepatocytes to transform into progenitor oval cells through secretory exosomes. The progenitor oval cells supplement hepatocytes in liver regeneration [44]. It is controversial whether MSC-derived exosomes (MSC-Ex) play a role in the treatment of liver failure through oxidative stress $[45,46]$. Yan et al. indicated that MSC-Ex had an anti-oxidant effect; they administered MSC-Ex up to $16 \mathrm{mg} / \mathrm{kg}$ body weight through the tail vein to treat liver failure via anti-oxidation and anti-apoptosis. MSC-Ex provided hepatoprotection via anti-oxidation to reduce hepatocyte injury caused by $\mathrm{CCl}_{4}$ and hydrogen peroxide $\left(\mathrm{H}_{2} \mathrm{O}_{2}\right)$ in vitro and in vivo and this process may be mediated by release of glutathione peroxidase-1 (GPX1) to reduce hepatic reactive oxygen species (ROS) and inhibit oxidative stress-induced apoptosis by upregulating ERK1/2 and Bcl-2 and suppressing the IKKB/ NFkB/casp-9/-3 pathway [45]. In contrast, another study showed that MSC-Ex inhibited acetaminophen (APAP) and $\mathrm{H}_{2} \mathrm{O}_{2}$ induced hepatocytes apoptosis by upregulating $\mathrm{Bcl}_{-\mathrm{xL}}$ protein and promoting the proliferation of hepatocytes. However, MSC-Ex cannot alleviate hepatocyte injury by regulating oxidative stress [46].

\section{Preclinical studies on MSC therapy for liver failure}

Many previous studies have shown that MSC transplantation can improve liver function, inhibit hepatocyte apoptosis, and promote hepatocyte proliferation in animal models of ALF [19, 36, 47-51]. Cai et al. established that BM-MSC transplantation decreased ALT and AST levels, downregulated Bax protein, and increased Bcl-2 expression compared with an acute liver injury (ALI) model [49]. In addition, MSC transplantation in rats can regulate liver and blood metabolic disorders, such as the imbalance of amino acids, bile acids, sphingolipids, acylcarnitines, and glycerophospholipids, which would increase proliferation and decrease apoptosis in hepatocytes $[48,52]$. Salomone et al. showed that AT-MSC transplantation in rats with ALI decreased AST, ALT, and prothrombin time (PT) and reduced liver isoprostanes, 8-hydroxyguanosine, and nitrite-nitrate levels but maintained glutathione levels. TNF- $\alpha$, MCP-1, IL-1 $\beta$, ICAM-1, and phospho-JNK levels in liver tissue after AT-MSC therapy did not increase significantly [51]. Meanwhile, AT-MSC transplantation remarkably improved the survival of ALF mice and reduced the severity of APAP-induced liver injury by inhibiting cytochrome P450 activity to decrease the accumulation of toxic nitrotyrosine and upregulation of NF-E2-related factor 2, resulting in an increase in anti-oxidant activity. These effects protected hepatocytes against APAPinduced injury by inhibiting the activation of MAPK signaling pathways and the production of inflammatory cytokines [53]. It has been reported that BM-MSCs play a therapeutic role in the pathogenesis of FHF and chronic fibrosis in mice by acting on various cells such as stimulating proliferation and inhibiting apoptosis of hepatocytes, reducing infiltrating macrophages, transforming $\mathrm{CD}^{+} \mathrm{T}$ lymphocytes into an anti-inflammatory state, and causing death of hepatic stellate cells [36]. Furthermore, BM-MSCs suppressed ConA-induced inflammatory responses to relieve liver damage by downregulating TNF-a, IFN- $\gamma$, and FasL and upregulating IL-10 mRNA [54]. IL-10 has the potential to treat ALF and exert an anti-inflammatory effect through activation of STAT3 signaling pathway and reducing NLRP3-caspase-1 inflammasome levels [42, 55]. In another study, BM-MSC transplantation significantly increased the survival time of pigs with FHF. The treatment group displayed a survival time longer than $14 \mathrm{~d}$ compared with the average survival time of $3.22 \mathrm{~d}$ in the control group. Analysis of cytokine arrays and metabolite profiles indicated that BM-MSC transplantation inhibited the life-threatening cytokine storm induced by D-GalN and stabilized FHF in pigs within $7 \mathrm{~d}$. Meanwhile, Delta-like ligand 4 was proven to support liver restoration in a pig FHF model [56]. Liu et al. proved that the therapeutic effect of intravenously injected UC-MSCs on reducing hepatocyte apoptosis and enhancing liver regeneration was mediated by paracrine pathways, involving the reduction of anti-oxidants (glutathione and superoxide dismutase), inflammatory factors (TNF- $\alpha$ and IL-6), and the increase of serum HGF levels [57]. Tonsil-derived MSCs (T-MSCs) express galectin-1, -3, -8, and -9; however, expression of galectin- 1 and -3 is more prominent than that of other galectins. Galectin-1 knockout reduces the immunosuppressive effect of T-MSCs on $\mathrm{CD} 4^{+} \mathrm{T}$ cells [58]. After galectin-1 knockout, $\mathrm{CD}^{+}$and $\mathrm{CD} 8^{+}$ T-cell proliferation recovered partially. However, the effect of MSCs on NK cells was unaffected by the downregulation of galectin-1. MSC-derived galectin-1 significantly regulated the release of cytokines, including TNF- $\alpha$, IFN- $\gamma$, IL-2, and IL-10 [59]. In D-Gal/LPS-induced ALF rats, BM-MSCs could significantly inhibit the nuclear factor-kappa B (NF-kB) pathway and reduce the levels of inflammatory factors, including IL-1 $\beta$, IL-6, and TNF- $\alpha$, by upregulating heme oxygenase- 1 (HO-1) [60]. Zheng et al. found that the high-mobility group box 1 protein (HMGB1) in serum and liver tissues is positively associated with liver damage. BM-MSC transplantation can improve liver function and liver pathology in ALF rats and reduce serum and liver HMGB1 [61]. Taken together, these preclinical studies clearly 
demonstrate that MSCs can effectively treat liver failure and explain the potential treatment mechanisms.

\section{Clinical trials of MSCs in the treatment of liver failure}

At present, although there are fewer clinical studies on MSC therapy for liver failure, previous studies have consistently agreed that MSCs can effectively treat liver failure. In an open-label randomized controlled study, allogeneic BM-MSC transplantation reduced serum TBIL and model for end-stage liver disease (MELD) scores in 56 patients with hepatitis B virus (HBV)-related acute-on-chronic liver failure (ACLF) that were injected with about 1 to $10 \times 10^{5}$ cells $/ \mathrm{kg}$ weekly for 4 weeks and followed up for 24 weeks. Meanwhile, BM-MSCs can reduce the incidence of serious infection and increase the cumulative survival rate. After treatment with BM-MSCs, no tumors were detected in any trial patients; however, fever occurred more frequently [62]. In a 24-month prospective study, the researchers used a single infusion of $100 \times 10^{6}$ UC-MSCs through the hepatic artery to treat 11 patients with HBV-related ACLF. In the treatment group, serum ALB, ALT, AST, bilirubin, direct bilirubin, $\mathrm{PT}$, international standardized ratio (INR), and MELD scores were significantly improved after 4 weeks of UC-MSC transplantation, and levels of ALB, PT, and INR also increased significantly at 24 months [63]. In a clinical trial conducted by Shi et al., UC-MSCs $\left(0.5 \times 10^{6}\right.$ cells $\left./ \mathrm{kg}\right)$ were intravenously infused three times at 4-week intervals to assess their therapeutic effects in 20 patients with HBV-associated ACLF. UC-MSC infusion significantly increased serum ALB, cholinesterase, prothrombin activity, platelet counts, and survival rate of patients with ACLF and decreased TBIL, ALT levels, and MELD scores [64]. Autologous BMMSC transplantation through the hepatic artery is safe for patients with HBV-associated liver failure. Shortterm outcomes are favorable; however, long-term outcomes have not improved significantly. The levels of ALB, TBIL, PT, and MELD scores in the transplantation group were significantly improved at about 2 to 3 weeks after transplantation; however, incidence of hepatocellular carcinoma or mortality did not differ significantly between the BM-MSC transplantation group and the control group after 192 weeks of follow-up [65].

\section{Exploring the best method of MSC therapy for liver failure}

Although many studies have confirmed the effectiveness of MSCs in treating liver failure, there is no standard protocol for MSC therapy. Numerous issues need further investigation regarding MSC treatment for liver failure, including selection of the optimal transplantation route, therapeutic effects of MSCs from different sources, and MSC colonization in vivo.
The principal methods involved in MSC transplantation include the peripheral, portal, and splenic veins; hepatic artery; intrahepatic injection; and intrasplenic injection, which may have different therapeutic effects in liver failure. Several studies suggested that administration via the portal vein had better results compared with other transplantation routes. Portal vein injection was superior to other MSC transplantation methods, including the hepatic artery, peripheral vein, and intrahepatic injection. Intraportal MSC injection could improve liver function, inhibit cell apoptosis, and prolong the survival time of pigs with ALF [66]. Similarly, MSCs via portal vein transplantation had better capability to reduce liver inflammation, decrease liver degeneration and necrosis, and promote liver regeneration compared with peripheral vein administration [67]. However, another study suggested that the liver function of rats with liver failure was significantly improved after MSC transplantation via three different routes, namely the hepatic artery, portal vein, and peripheral vein, but no significant difference was observed among the three groups [68]. There was no significant difference in liver enzyme, albumin, bilirubin, and hemoglobin levels; total white blood cell count; and platelets in patients with liver failure treated via intrahepatic and intrasplenic injection of MSCs [47, 69]. In addition, UC-MSC transplantation through the peripheral vein displayed similar curative effects with intrahepatic injection [19]. After transplantation of AT-MSCs through the peripheral vein and the splenic vein, ALF was improved in pigs. Splenic vein transplantation has better therapeutic effects than peripheral vein transplantation in protecting liver function, reducing proinflammatory factors, increasing anti-inflammatory factors, and promoting liver regeneration [70].

Hepatic colonization of MSCs is one of the principal factors affecting MSC therapy for liver failure. Zhu et al. showed that BM-MSCs were detected in injured liver tissue at $24 \mathrm{~h}$ after transplantation, and no BM-MSCs were detected in mice without liver injury, suggesting that tissue injury could recruit BM-MSCs in vivo. All transplanted BM-MSCs located near the hepatic portal vein disappeared [33]. Furthermore, MSCs transplanted into the liver were related to the presence of liver injury $[20,71]$ and unrelated to the injection site $[18,72]$. In the current MSC transplantation, only a few MSCs can colonize the liver, and we can improve the hepatic colonizability by modifying MSCs. c-Met-MSCs significantly enhanced the homing ability to the injured liver and increased the survival rate and liver function in rats with liver failure [73]. A similar study demonstrated that MSCs expressing C-X-C chemokine receptor type 4 (CXCR4) had greater hepatic colonizability and contributed to restore the damaged liver [74, 75]. It has been reported that stromal cell-derived factor-1 (SDF-1), as a 
chemotactic factor, may promote the migration of MSCs to the liver through the SDF-1/CXCR4 axis and that SDF-1 mobilizing MSCs can enhance liver regeneration after liver injury [76].

It is unclear whether MSCs from different sources have the same therapeutic effects on liver failure. Compared with the results of biochemical analysis, histopathological assessment, gene expression of HLCs, and survival rate, AT-MSCs treated liver failure more effectively than BM-MSCs did [24, 77]. However, another study established that only BM-MSCs reduced liver damage and alleviated liver failure in ConA-induced mice, compared with mature hepatocytes, fetal liver cells, and induced hepatic stem cells [54]. Recently, it has been suggested that combined transplantation of MSCs and mature hepatocytes in ALF may be a good combination to facilitate liver repair and antiinflammatory effects [78, 79]. The liver failure microenvironment contributed to the transplanted MSCs to express hepatocyte-specific genes [80, 81]. Meanwhile, ALF upregulated liver-specific genes in MSCs but did not affect its stem cell characteristics and cell viability [80]. The serum from rats with ALF induced the expression of CXCR4 on MSCs, which enhanced the homing ability of MSCs to damaged liver tissue [75].

In addition to transplantation of MSCs alone, liver failure may be synergistically treated by enhancing growth-related gene expression or blocking the effect of inflammatory factors. Tang et al. reported that UCMSCs overexpressing HGF can reduce liver damage and prolong the survival rate of APAP-induced ALF mice through anti-apoptosis and anti-oxidation [82]. Wang et al. found that c-Met expression in hepatocytes is closely associated with HGF-mediated liver regeneration [73]. Hepatocytes could specifically express the c-Met gene through gene transfer in vivo, thus enhancing hepatocyte proliferation, decreasing apoptosis, and significantly improving overall survival rates [83]. It has been shown that IL-1Ra is a natural IL-1 antagonist, which can block the inflammatory process through competitive binding with IL-1 receptor [84]. Therefore, IL-1Ra could decrease IL-1, IL-6, TNF- $\alpha$, and other inflammatory markers in a short period [21, 85]. MSCs overexpressing IL-1Ra gene can be transplanted into the injured liver to improve liver function and survival rate in animal models of ALF [85]. Similar results showed that combined treatment of IL-1Ra and MSCs could promote the recovery in pigs with ALI and had better therapeutic effects than MSCs alone [86, 87].

\section{Conclusion}

MSCs have many properties, including immunoregulation, differentiation into HLCs, and repair of damaged tissue, which contribute to the treatment of liver failure. Many previous studies have shown that MSCs can effectively treat liver failure, most preclinical studies but few clinical studies. In most of the existing studies, researchers observed the short-term benefits of MSC therapy but long-term efficacy was lacking. Therefore, it is unclear whether MSC therapy can provide long-term benefits. The mechanism of MSC treatment for liver failure is primarily focused on differentiation of MSCs into hepatocytes and immunoregulation, and the effect of immunoregulation seems more obvious. In addition to MSC therapy alone, MSC modification or MSCs combined with other treatment methods are being increasingly considered. But there are still many problems to be solved in standardizing the process of MSC therapy for liver failure, including determination of the optimal time, dose, and route for MSC transplantation; improvement of the colonization rate and survival rate of MSCs in the liver; and the safety of MSC transplantation. Consequently, although MSCs have great potential in treating liver failure, they are facing numerous challenges prior to clinical application.

\section{Abbreviations}

ACLF: Acute-on-chronic liver failure; AF-MSC: Amniotic fluid mesenchymal stem cell; ALB: Albumin; ALF: Acute liver failure; ALI: Acute liver injury; ALT: Alanine transaminase; APAP: Acetaminophen; AST: Aspartate transaminase; AT-MSC: Adipose tissue mesenchymal stem cell; bFGF: Basic fibroblast growth factor; BM-MSC: Bone marrow mesenchymal stem cell; $\mathrm{CCl}_{4}$ : Carbon tetrachloride; CXCR4: C-X-C chemokine receptor type 4; D-GalN/ LPS: D-galactosamine/lipopolysaccharide; EGF: Epidermal growth factor; FasL: Fas ligand; FHF: Fulminant hepatic failure; $\mathrm{H}_{2} \mathrm{O}_{2}$ : Hydrogen peroxide; HBV: Hepatitis B virus; HGF: Hepatocyte growth factor; HLC: Hepatocyte-like cell; HMGB1: High-mobility group box 1; HPL-CM: Conditioned medium of hepatic progenitor-like cells; IDO: Indoleamine 2,3-oxidase; IFN-Y: Interferongamma; IL: Interleukin; IL-1Ra: Interleukin-1-receptor antagonist; INR: International standardized ratio; ITS: Insulin transferrin-selenium; MELD: Model for end-stage liver disease; MSC: Mesenchymal stem cell; MSCCM: Mesenchymal stem cell conditioned medium; MSC-Ex: Mesenchymal stem cell-derived exosomes; NK: Natural killer; OSM: Oncostatin M; PT: Prothrombin time; SDF-1: Stromal cell-derived factor-1; STAT3: Signal transducer and activator of transcription 3; TBIL: Total bilirubin; TGF$\beta$ : Transforming growth factor-beta; Th: T helper; T-MSC: Tonsil-derived mesenchymal stem cell; TNF-a: Tumor necrosis factor-alpha; Treg: Regulatory T; UC-MSC: Umbilical cord mesenchymal stem cell

\section{Acknowledgments \\ We thank Meng-Lan Wang and Ya-Chao Tao for their kind suggestions about the manuscript.}

\section{Availability of data and materials}

Data sharing is not applicable to this article as no datasets were generated or analyzed during the present study.

\section{Authors' contributions}

WYH drafted the manuscript and was a major contributor in writing the manuscript. WDB and $C B$ researched the references and participated in the writing of the manuscript. CEQ and TH conceived the idea and revised the manuscript. All authors read and approved the final manuscript.

Ethics approval and consent to participate Not applicable. 


\section{Consent for publication}

Not applicable.

\section{Competing interests}

The authors declare that they have no competing interests.

\section{Publisher's Note}

Springer Nature remains neutral with regard to jurisdictional claims in published maps and institutional affiliations.

\section{Published online: 24 August 2018}

\section{References}

1. Saliba F, Samuel D. Acute liver failure: current trends. J Hepatol. 2013;59:6-8.

2. Bernal W, Auzinger G, Dhawan A, Wendon J. Acute liver failure. Lancet. 2010;376:190-201

3. Volarevic V, Nurkovic J, Arsenijevic N, Stojkovic M. Concise review: therapeutic potential of mesenchymal stem cells for the treatment of acute liver failure and cirrhosis. Stem Cells. 2014;32:2818-23.

4. Tao YC, Wang ML, Chen EQ, Tang H. Stem cells transplantation in the treatment of patients with liver failure. Curr Stem Cell Res Ther. 2018;13: 193-201.

5. Wang J, Cen P, Chen J, Fan L, Li J, Cao H, et al. Role of mesenchymal stem cells, their derived factors, and extracellular vesicles in liver failure. Stem Cell Res Ther. 2017:8:137.

6. Liu WH, Song FQ, Ren LN, Guo WQ, Wang T, Feng YX, et al. The multiple functional roles of mesenchymal stem cells in participating in treating liver diseases. J Cell Mol Med. 2015;19:511-20.

7. Huebert RC, Rakela J. Cellular therapy for liver disease. Mayo Clin Proc. 2014; 89:414-24.

8. Ferrer JR, Chokechanachaisakul A, Wertheim JA. New tools in experimental cellular therapy for the treatment of liver diseases. Curr Transplant Rep. 2015:2:202-10.

9. Aurich I, Mueller LP, Aurich H, Luetzkendorf J, Tisljar K, Dollinger MM, et al. Functional integration of hepatocytes derived from human mesenchymal stem cells into mouse livers. Gut. 2007;56:405-15.

10. Lee KD, Kuo TK, Whang-Peng J, Chung YF, Lin CT, Chou SH, et al. In vitro hepatic differentiation of human mesenchymal stem cells. Hepatology. 2004:40:1275-84.

11. Banas A, Teratani T, Yamamoto Y, Tokuhara M, Takeshita F, Quinn G, et al. Adipose tissue-derived mesenchymal stem cells as a source of human hepatocytes. Hepatology. 2007:46:219-28.

12. Zagoura DS, Roubelakis MG, Bitsika V, Trohatou O, Pappa KI, Kapelouzou A, et al. Therapeutic potential of a distinct population of human amniotic fluid mesenchymal stem cells and their secreted molecules in mice with acute hepatic failure. Gut. 2012;61:894-906.

13. Stock P, Bruckner S, Ebensing S, Hempel M, Dollinger MM, Christ B. The generation of hepatocytes from mesenchymal stem cells and engraftment into murine liver. Nat Protoc. 2010;5:617-27.

14. Yamazaki S, Miki K, Hasegawa K, Sata M, Takayama T, Makuuchi M. Sera from liver failure patients and a demethylating agent stimulate transdifferentiation of murine bone marrow cells into hepatocytes in coculture with nonparenchymal liver cells. J Hepatol. 2003;39:17-23.

15. Shapiro RM, Lazo-Langner A. Systematic review of azacitidine regimens in myelodysplastic syndrome and acute myeloid leukemia. BMC Hematol. 2018;18:3

16. Muller A, Florek M. 5-Azacytidine/Azacitidine. Recent results in cancer research. Fortschritte der Krebsforschung. Progres dans les recherches sur le cancer. 2010;184:159-70.

17. Yang JF, Cao HC, Pan QL, Yu J, Li J, Li LJ. Mesenchymal stem cells from the human umbilical cord ameliorate fulminant hepatic failure and increase survival in mice. Hepatobiliary Pancreat Dis Int. 2015;14:186-93.

18. Yu J, Cao H, Yang J, Pan Q, Ma J, Li J, et al. In vivo hepatic differentiation of mesenchymal stem cells from human umbilical cord blood after transplantation into mice with liver injury. Biochem Biophys Res Commun. 2012;422:539-45.

19. Zheng S, Yang J, Yang J, Tang Y, Shao Q, Guo L, et al. Transplantation of umbilical cord mesenchymal stem cells via different routes in rats with acute liver failure. Int J Clin Exp Pathol. 2015;8:15854-62.
20. Shi LL, Liu FP, Wang DW. Transplantation of human umbilical cord blood mesenchymal stem cells improves survival rates in a rat model of acute hepatic necrosis. Am J Med Sci. 2011;342:212-7.

21. Xiao JQ, Shi XL, Ma HC, Tan JJ, Lin Z, Xu Q, et al. Administration of IL-1Ra chitosan nanoparticles enhances the therapeutic efficacy of mesenchymal stem cell transplantation in acute liver failure. Arch Med Res. 2013:44:370-9.

22. Chen G, Jin Y, Shi X, Qiu Y, Zhang Y, Cheng M, et al. Adipose-derived stem cell-based treatment for acute liver failure. Stem Cell Res Ther. 2015;6:40.

23. Wang H, Zhao T, Xu F, Li Y, Wu M, Zhu D, et al. How important is differentiation in the therapeutic effect of mesenchymal stromal cells in liver disease? Cytotherapy. 2014;16:309-18.

24. Manzini BM, da Silva Santos Duarte A, Sankaramanivel S, Ramos AL, LatufFilho P, Escanhoela C, et al. Useful properties of undifferentiated mesenchymal stromal cells and adipose tissue as the source in liverregenerative therapy studied in an animal model of severe acute fulminant hepatitis. Cytotherapy. 2015;17:1052-65.

25. Li D, Fan J, He X, Zhang X, Zhang Z, Zeng Z, et al. Therapeutic effect comparison of hepatocyte-like cells and bone marrow mesenchymal stem cells in acute liver failure of rats. Int J Clin Exp Pathol. 2015;8:11-24.

26. Zhou R, Li Z, He C, Li R, Xia H, Li C, et al. Human umbilical cord mesenchymal stem cells and derived hepatocyte-like cells exhibit similar therapeutic effects on an acute liver failure mouse model. PLoS One. 2014;9: e104392.

27. Su Z, Li P, Wu B, Ma H, Wang Y, Liu G, et al. PHBVHHx scaffolds loaded with umbilical cord-derived mesenchymal stem cells or hepatocyte-like cells differentiated from these cells for liver tissue engineering. Mater Sci Eng C Mater Biol Appl. 2014;45:374-82.

28. Akiyama K, Chen C, Wang D, Xu X, Qu C, Yamaza T, et al. Mesenchymalstem-cell-induced immunoregulation involves FAS-ligand-/FAS-mediated T cell apoptosis. Cell Stem Cell. 2012;10:544-55.

29. Fang $X$, Liu L, Dong J, Zhang J, Song $H$, Song $Y$, et al. A study about immunomodulatory effect and efficacy and prognosis of human umbilical cord mesenchymal stem cells in patients with chronic hepatitis B-induced decompensated liver cirrhosis. J Gastroenterol Hepatol. 2018;33:774-80.

30. Zhang Y, Cai W, Huang Q, Gu Y, Shi Y, Huang J, et al. Mesenchymal stem cells alleviate bacteria-induced liver injury in mice by inducing regulatory dendritic cells. Hepatology. 2014;59:671-82.

31. Corcione A, Benvenuto F, Ferretti E, Giunti D, Cappiello V, Cazzanti F, et al. Human mesenchymal stem cells modulate B-cell functions. Blood. 2006;107: 367-72.

32. Milosavljevic N, Gazdic M, Simovic Markovic B, Arsenijevic A, Nurkovic J, Dolicanin Z, et al. Mesenchymal stem cells attenuate acute liver injury by altering ratio between interleukin 17 producing and regulatory natural killer T cells. Liver Transpl. 2017;23:1040-50.

33. Zhu X, He B, Zhou X, Ren J. Effects of transplanted bone-marrow-derived mesenchymal stem cells in animal models of acute hepatitis. Cell Tissue Res. 2013:351:477-86.

34. Border WA, Noble NA. Transforming growth factor beta in tissue fibrosis. N Engl J Med. 1994;331:1286-92.

35. Bataller R, Brenner DA. Liver fibrosis. J Clin Invest. 2005;115:209-18.

36. Huang $B$, Cheng X, Wang H, Huang W, la Ga HZ, Wang D, et al. Mesenchymal stem cells and their secreted molecules predominantly ameliorate fulminant hepatic failure and chronic liver fibrosis in mice respectively. J Transl Med. 2016;14:45.

37. Zhao X, Shi X, Zhang Z, Ma H, Yuan X, Ding Y. Combined treatment with MSC transplantation and neutrophil depletion ameliorates D-GalN/LPSinduced acute liver failure in rats. Clin Res Hepatol Gastroenterol. 2016;40: 730-8.

38. Lotfinia M, Kadivar M, Piryaei A, Pournasr B, Sardari S, Sodeifi N, et al Effect of secreted molecules of human embryonic stem cell-derived mesenchymal stem cells on acute hepatic failure model. Stem Cells Dev. 2016;25:1898-908.

39. van Poll D, Parekkadan B, Cho CH, Berthiaume F, Nahmias Y, Tilles AW, et al. Mesenchymal stem cell-derived molecules directly modulate hepatocellular death and regeneration in vitro and in vivo. Hepatology. 2008;47:1634-43.

40. Parekkadan B, van Poll D, Suganuma K, Carter EA, Berthiaume F, Tilles AW, et al. Mesenchymal stem cell-derived molecules reverse fulminant hepatic failure. PLoS One. 2007:2:e941.

41. Herrera MB, Fonsato V, Bruno S, Grange C, Gilbo N, Romagnoli R, et al. Human liver stem cells improve liver injury in a model of fulminant liver failure. Hepatology. 2013;57:311-9. 
42. Ma HC, Wang X, Wu MN, Zhao X, Yuan XW, Shi XL. Interleukin-10 Contributes to Therapeutic Effect of Mesenchymal Stem Cells for Acute Liver Failure via Signal Transducer and Activator of Transcription 3 Signaling Pathway. Chin Med J (Engl). 2016;129:967-75.

43. Chen L, Xiang B, Wang X, Xiang C. Exosomes derived from human menstrual blood-derived stem cells alleviate fulminant hepatic failure. Stem Cell Res Ther. 2017:8:9.

44. Wu HH, Lee OK. Exosomes from mesenchymal stem cells induce the conversion of hepatocytes into progenitor oval cells. Stem Cell Res Ther 2017:8:117.

45. Yan Y, Jiang W, Tan Y, Zou S, Zhang H, Mao F, et al. hucMSC ExosomeDerived GPX1 Is Required for the Recovery of Hepatic Oxidant Injury. Mol Ther. 2017;25:465-79.

46. Tan CY, Lai RC, Wong W, Dan YY, Lim SK, Ho HK. Mesenchymal stem cellderived exosomes promote hepatic regeneration in drug-induced liver injury models. Stem Cell Res Ther. 2014;5:76.

47. Gilsanz C, Aller MA, Fuentes-Julian S, Prieto I, Blazquez-Martinez A, Argudo S, et al. Adipose-derived mesenchymal stem cells slow disease progression of acute-on-chronic liver failure. Biomed Pharmacother. 2017:91:776-87.

48. Tautenhahn HM, Bruckner S, Baumann S, Winkler S, Otto W, von Bergen M, et al. Attenuation of postoperative acute liver failure by mesenchymal stem cell treatment due to metabolic implications. Ann Surg. 2016;263:546-56.

49. Cai Y, Zou Z, Liu L, Chen S, Chen Y, Lin Z, et al. Bone marrow-derived mesenchymal stem cells inhibits hepatocyte apoptosis after acute liver injury. Int J Clin Exp Pathol. 2015;8:107-16.

50. Yuan $S$, Jiang $T$, Sun $L$, Zheng $R$, Ahat N, Zhang $Y$. The role of bone marrow mesenchymal stem cells in the treatment of acute liver failure. Biomed Res Int. 2013;2013:251846.

51. Salomone F, Barbagallo I, Puzzo L, Piazza C, Li VG. Efficacy of adipose tissuemesenchymal stem cell transplantation in rats with acetaminophen liver injury. Stem Cell Res. 2013;11:1037-44.

52. Li J, Xin J, Hao S, Zhang L, Jiang L, Chen D, et al. Return of the metabolic trajectory to the original area after human bone marrow mesenchymal stem cell transplantation for the treatment of fulminant hepatic failure. Proteome Res. 2012;11:3414-22.

53. Huang YJ, Chen P, Lee CY, Yang SY, Lin MT, Lee HS, et al. Protection against acetaminophen-induced acute liver failure by omentum adipose tissue derived stem cells through the mediation of Nrf2 and cytochrome P450 expression. J Biomed Sci. 2016;23:5.

54. Sun $\mathrm{K}$, Xie X, Xie J, Jiao S, Chen X, Zhao X, et al. Cell-based therapy for acute and chronic liver failures: distinct diseases, different choices. Sci Rep. 2014;4:6494.

55. Wang J, Ren H, Yuan X, Ma H, Shi X, Ding Y. Interleukin-10 secreted by mesenchymal stem cells attenuates acute liver failure through inhibiting pyroptosis. Hepatol Res. 2018;48:E194-202.

56. Shi D, Zhang J, Zhou Q, Xin J, Jiang J, Jiang L, et al. Quantitative evaluation of human bone mesenchymal stem cells rescuing fulminant hepatic failure in pigs. Gut. 2017;66:955-64.

57. Liu Z, Meng F, Li C, Zhou X, Zeng X, He Y, et al. Human umbilical cord mesenchymal stromal cells rescue mice from acetaminophen-induced acute liver failure. Cytotherapy. 2014;16:1207-19.

58. Ryu KH, Kim SY, Kim YR, Woo SY, Sung SH, Kim HS, et al. Tonsil-derived mesenchymal stem cells alleviate concanavalin A-induced acute liver injury. Exp Cell Res. 2014;326:143-54.

59. Gieseke F, Bohringer J, Bussolari R, Dominici M, Handgretinger R, Muller I. Human multipotent mesenchymal stromal cells use galectin-1 to inhibit immune effector cells. Blood. 2010;116:3770-9.

60. Zhang ZH, Zhu W, Ren HZ, Zhao X, Wang S, Ma HC, et al. Mesenchymal stem cells increase expression of heme oxygenase-1 leading to anti-inflammatory activity in treatment of acute liver failure. Stem Cell Res Ther. 2017:8:70.

61. Zheng S, Yang J, Tang Y, Yang J, Shao Q, Guo L, et al. Effect of bone marrow mesenchymal stem cells transplantation on the serum and liver HMGB1 expression in rats with acute liver failure. Int J Clin Exp Pathol. 2015; 8:15985-92.

62. Lin BL, Chen JF, Qiu WH, Wang KW, Xie DY, Chen XY, et al. Allogeneic bone marrow-derived mesenchymal stromal cells for hepatitis B virus-related acute-on-chronic liver failure: a randomized controlled trial. Hepatology. 2017;66:209-19.

63. Li YH, Xu Y, Wu HM, Yang J, Yang LH, Yue-Meng W. Umbilical cord-derived mesenchymal stem cell transplantation in hepatitis B virus related acute-onchronic liver failure treated with plasma exchange and Entecavir: a 24month prospective study. Stem Cell Rev. 2016;12:645-53.
64. Shi M, Zhang Z, Xu R, Lin H, Fu J, Zou Z, et al. Human mesenchymal stem cell transfusion is safe and improves liver function in acute-on-chronic liver failure patients. Stem Cells Transl Med. 2012;1:725-31.

65. Peng L, Xie DY, Lin BL, Liu J, Zhu HP, Xie C, et al. Autologous bone marrow mesenchymal stem cell transplantation in liver failure patients caused by hepatitis B: short-term and long-term outcomes. Hepatology. 2011;54:820-8.

66. Sang JF, Shi XL, Han B, Huang T, Huang X, Ren HZ, et al. Intraportal mesenchymal stem cell transplantation prevents acute liver failure through promoting cell proliferation and inhibiting apoptosis. Hepatobiliary Pancreat Dis Int. 2016;15:602-11.

67. Cao H, Yang J, Yu J, Pan Q, Li J, Zhou P, et al. Therapeutic potential of transplanted placental mesenchymal stem cells in treating Chinese miniature pigs with acute liver failure. BMC Med. 2012;10:56.

68. Sun L, Fan X, Zhang L, Shi G, Aili M, Lu X, et al. Bone mesenchymal stem cell transplantation via four routes for the treatment of acute liver failure in rats. Int J Mol Med. 2014;34:987-96.

69. Amer ME, El-Sayed SZ, El-Kheir WA, Gabr H, Gomaa AA, El-Noomani N, et al. Clinical and laboratory evaluation of patients with end-stage liver cell failure injected with bone marrow-derived hepatocyte-like cells. Eur J Gastroenterol Hepatol. 2011;23:936-41.

70. Teshima T, Matsumoto $H$, Michishita M, Matsuoka A, Shiba M, Nagashima T, et al. Allogenic adipose tissue-derived mesenchymal stem cells ameliorate acute hepatic injury in dogs. Stem Cells Int. 2017;2017: 3892514.

71. Li J, Zhang L, Xin J, Jiang L, Li J, Zhang T, et al. Immediate intraportal transplantation of human bone marrow mesenchymal stem cells prevents death from fulminant hepatic failure in pigs. Hepatology. 2012;56:1044-52.

72. Maijenburg MW, van der Schoot CE, Voermans C. Mesenchymal stromal cell migration: possibilities to improve cellular therapy. Stem Cells Dev. 2012;21:19-29.

73. Wang K, Li Y, Zhu T, Zhang Y, Li W, Lin W, et al. Overexpression of c-met in bone marrow mesenchymal stem cells improves their effectiveness in homing and repair of acute liver failure. Stem Cell Res Ther. 2017:8:162.

74. Ma HC, Shi XL, Ren HZ, Yuan XW, Ding YT. Targeted migration of mesenchymal stem cells modified with CXCR4 to acute failing liver improves liver regeneration. World J Gastroenterol. 2014;20:14884-94.

75. Deng C, Qin A, Zhao W, Feng T, Shi C, Liu T. Up-regulation of CXCR4 in rat umbilical mesenchymal stem cells induced by serum from rat with acute liver failure promotes stem cells migration to injured liver tissue. Mol Cell Biochem. 2014;396:107-16.

76. Jin SZ, Meng XW, Han MZ, Sun X, Sun LY, Liu BR. Stromal cell derived factor-1 enhances bone marrow mononuclear cell migration in mice with acute liver failure. World J Gastroenterol. 2009;15:2657-64.

77. Zare H, Jamshidi S, Dehghan MM, Saheli M, Piryaei A. Bone marrow or adipose tissue mesenchymal stem cells: comparison of the therapeutic potentials in mice model of acute liver failure. J Cell Biochem. 2018;119(7):5834-42.

78. Fitzpatrick E, Wu Y, Dhadda P, Hughes RD, Mitry RR, Qin H, et al. Coculture with mesenchymal stem cells results in improved viability and function of human hepatocytes. Cell Transplant. 2015;24:73-83.

79. Liu M, Yang J, Hu W, Zhang S, Wang Y. Superior performance of co-cultured mesenchymal stem cells and hepatocytes in poly(lactic acid-glycolic acid) scaffolds for the treatment of acute liver failure. Biomed Mater. 2016;11:015008.

80. Hu C, Zhou N, Li J, Shi D, Cao H, Li J, et al. Porcine adipose-derived mesenchymal stem cells retain their stem cell characteristics and cell activities while enhancing the expression of liver-specific genes after acute liver failure. Int J Mol Sci. 2016;17(1).

81. Li J, Tao R, Wu W, Cao H, Xin J, Guo J, et al. Transcriptional profiling and hepatogenic potential of acute hepatic failure-derived bone marrow mesenchymal stem cells. Differentiation. 2010;80:166-74.

82. Tang Y, Li Q, Meng F, Huang X, Li C, Zhou X, et al. Therapeutic potential of HGF-expressing human umbilical cord mesenchymal stem cells in mice with acute liver failure. Int J Hepatol. 2016;2016:5452487.

83. Zhu C, Li Y, Li W, Wu Q, Gao R. Gene transfer of c-met confers protection against D-galactosamine/lipopolysaccharide-induced acute liver failure. Dig Dis Sci. 2012;57:925-34.

84. Volarevic V, Al-Qahtani A, Arsenijevic N, Pajovic S, Lukic ML. Interleukin-1 receptor antagonist (IL-1Ra) and IL-1Ra producing mesenchymal stem cells as modulators of diabetogenesis. Autoimmunity. 2010;43:255-63.

85. Zheng YB, Zhang XH, Huang ZL, Lin CS, Lai J, Gu YR, et al. Amnioticfluid-derived mesenchymal stem cells overexpressing interleukin-1 receptor antagonist improve fulminant hepatic failure. PLoS One. 2012; 7:e41392 
86. Shi XL, Zhu W, Tan JJ, Xiao JQ, Zhang L, Xu Q, et al. Effect evaluation of interleukin-1 receptor antagonist nanoparticles for mesenchymal stem cell transplantation. World J Gastroenterol. 2013;19:1984-91.

87. Ma H, Shi X, Yuan X, Ding Y. IL-1 beta siRNA adenovirus benefits liver

regeneration by improving mesenchymal stem cells survival after acute liver failure. Ann Hepatol. 2016;15:260-70. 